\title{
THE UNIFORMIZATION OF A HYPERBOLIC RIEMANN SURFACE
}

\section{GEORGE INNIS}

In this paper a hyperbolic Riemann surface is described, and the uniformizing function for this surface is constructed. The uniformizing function for this hyperbolic surface is an infinite product similar to the ones constructed for some parabolic surfaces in earlier studies [1], [2], [4].

Description of the surface. Let $I$ denote the positive integers. For each $i \in I$, let $C_{i}$ be the vertical line segment in the $Z$-plane of length 2 centered at the point $(1 / i, 0)$. Let $S_{1}$ be the $Z$-sphere cut along $C_{1}$ and for each $i \in I, i>1$, let $S_{i}$ be the $Z$-sphere cut along $C_{i-1}$ and along $C_{i}$. For each $i \in I$, join $S_{i}$ to $S_{i+1}$ along $C_{i}$ in such a way as to form first-order branch points over $(1 / i, 1)$ and $(1 / i,-1)$. The resulting Riemann surface $F$ is simply connected and is easily seen to be hyperbolic because the surface has a free edge along the vertical line segment from $-i$ to $i$.

Approximating surfaces. For each $i \in I$, let $F_{i}$ be the Riemann surface formed from the first $i$ sheets of $F$ with the points of the curve $C_{i}$ on $S_{i}$ deleted. Let $F_{i}^{*}$ be the closed surface formed from the first $i$ sheets of $F$ with $C_{i}$ on $S_{i}$ healed. There exists a unique function $g_{i}$ mapping $F_{i}^{*}$ onto the $Z$-sphere such that $f_{i}=g_{i}^{-1}, f_{i}(0)=0 \in S_{1}$, $f_{i}^{\prime}(0)=1$, and $f_{i}(\infty)=\infty \in S_{i}$. Let $g$ be the unique function mapping $F$ onto the disk $\{|z|<R \leqq \infty\}$ such that if $f=g^{-1}$, then $f(0)=0 \in S_{1}$ and $f^{\prime}(0)=1$.

LEMMA 1. $f_{i}$ is real for real $z$.

Proof. The proof of this lemma is similar to the arguments of earlier authors [1], [4].

$g_{i}\left(F_{i}\right)$ is a simply connected domain of the $Z$-sphere with at least two boundary points (all points of $g_{i}\left(C_{i}\right)$ are boundary points). On removing the real axis from $g_{i}\left(F_{i}\right)$, the portion of $g_{i}\left(F_{i}\right)$ in the upper half plane is simply connected and open and, hence, can be mapped one-one and conformally onto a half disk $\{z:|z|<1, \operatorname{Im} z>0\}$ in such a way that the real axis corresponds to a portion of the real axis and the points of $g_{i}\left(C_{i}\right)$ correspond to points of the semicircle. The symmetry principle applies to assure the existence of a function $h_{i}$ such that $h_{i}\left(g_{i}\left(F_{i}\right)\right)$ is a disk with center at 0 and points of the real axis correspond to points of the real diagonal of the disk. Furthermore, we

Received by the editors June 7, 1965. 
can normalize $h_{i}$ (call it $h_{i}$ still) so that $h_{i}(0)=0$ and $h_{i}^{\prime}(0)=1$. Then $h_{i}\left(g_{i}\left(F_{i}\right)\right)$ is a disk of radius $r_{i}>0$ about 0 .

Notation. Let the origin on $S_{i}$ be denoted by $0_{i}$ and the point at $\infty$ on $S_{i}$ by $\infty_{i}$. Also let

$$
\begin{aligned}
\delta_{k, i} & =g_{i}\left(0_{k}\right), & \delta_{k} & =g\left(0_{k}\right), \\
\gamma_{k, i} & =g_{i}\left(\infty_{k}\right), & \gamma_{k} & =g\left(\infty_{k}\right), \\
\delta_{k, i}^{*} & =1-\frac{z}{\delta_{k, i}}, & \delta_{k}^{*} & =1-\frac{z}{\delta_{k}}, \\
\gamma_{k, i}^{*} & =1-\frac{z}{\gamma_{k, i}}, & \gamma_{k}^{*} & =1-\frac{z}{\gamma_{k}} .
\end{aligned}
$$

LEMma 2.

$$
R_{i}(z)=\frac{z}{\gamma_{1, i}^{*}} \prod_{k=2}^{i} \frac{\delta_{k, i}^{*}}{\gamma_{k, i}^{*}}
$$

maps $\left\{|z|<r_{i}\right\}$ one-one and conformally onto $F_{i}$.

ProOF. $F_{i}^{*}$ is a closed simply connected Riemann surface and is, therefore, the surface of the inverse of a unique rational function $Q_{i}$ such that $Q_{i}(0)=0_{1}, Q_{i}^{\prime}(0)=1$, and $Q_{i}(\infty)=\infty_{i}$. Composing $Q_{i}$ with $h_{i}^{-1}$ yields a unique rational function $R_{i}$ mapping $\left\{|z|<r_{i}\right\}$ one-one and conformally on to $F_{i}$ such that $R_{i}(0)=0$, and $R_{i}^{\prime}(0)=1$.

Lemma 3. $R_{i}(z) \rightarrow f(z)$ uniformly on compact subsets of $\{|z|<r\}$ for some $r>0$.

Proof. Let $D_{i}=\left\{|z|<r_{i}\right\}$. This result then follows immediately by applying MacLane's theorem [3, p. 33] with Corollary $\alpha$ to the two sequences of surfaces $\left\{F_{i}, 0\right\}$ and $\left\{D_{i}, 0\right\}$. We also get that $r_{i} \rightarrow r>0$.

LEMMA 4. $\delta_{k, i} \rightarrow \delta_{k}$ and $\gamma_{k, i} \rightarrow \gamma_{k}$ as $i \rightarrow \infty$.

Proof. This is a consequence of Hurewitz theorem or follows as one of the conclusions of the theorem of MacLane cited in the proof of Lemma 3.

Lemma 5. The infinite product

$$
\pi(z)=\frac{z}{\gamma_{1}^{*}} \prod_{k=2}^{\infty} \frac{\delta_{k}^{*}}{\gamma_{k}^{*}}
$$

converges subuniformly on $\{|z|<r\}$. 
PRoOF. Let $r_{0} \leqq r$. Only a finite number of the $\delta_{k}$ or $\gamma_{k}$ lie in $\left\{|z|<r_{0}\right\}$ and thus there exists $n_{0}>0$ such that for $k>n_{0}, \delta_{k}$ and $\gamma_{k}$ lie in the ring $r_{0}<|z|<r$. Since for each $k \in I, \delta_{k}>\gamma_{k}$, there exists $\epsilon_{k}>0$ such that $\gamma_{k}+\epsilon_{k}=\delta_{k}$. Because of the location of $\gamma_{k}$ and $\delta_{k}, \sum_{k=1}^{\infty} \epsilon_{k}$ converges to some number less than $2 r$. To prove the subuniform convergence of $\pi$, it is sufficient to prove the uniform convergence of

$$
\sum_{2}^{\infty} \log \frac{\delta_{k}^{*}}{\gamma_{k}^{*}}
$$

for $z \in\left\{|z| \leqq r_{0}\right\}$. By the Cauchy criterion, this last series converges uniformly in $\left\{|z| \leqq r_{0}\right\}$ provided that for each $\epsilon>0$ and for all $z$ with $|z| \leqq r_{0}$, there exists a number $N(\epsilon)>0$ such that for $n>N(\epsilon)$ and for all $p>0$,

$$
\left|\sum_{k=n_{0}+n}^{n_{0}+n+p} \log \frac{\delta_{k}^{*}}{\gamma_{k}^{*}}\right|<\epsilon
$$

Now

$$
\begin{aligned}
\left|\sum_{k=n_{0}+n}^{n++n+p} \log \frac{\delta_{k}^{*}}{\gamma_{k}^{*}}\right| & =\left|\sum_{m=1}^{\infty} \frac{z^{m}}{m} \sum_{k=n_{0}+n}^{n_{0}+n+p}\left(\frac{1}{\gamma_{k}^{m}}-\frac{1}{\delta_{k}^{m}}\right)\right| \\
& =\left|\sum_{m=1}^{\infty} \frac{z^{m}}{m} \sum_{k=n_{0}+n}^{n_{0}+n+p} \frac{\delta_{k}^{m}-\gamma_{k}^{m}}{\gamma_{k}^{m} \delta_{k}^{m}}\right| \\
& \leqq\left|\sum_{m=1}^{\infty} \frac{z^{m}}{m \gamma_{n_{0}+n}^{m}} \sum_{k=n_{0}+n}^{n_{0}+n+p} 1-\left(\frac{\gamma_{k}}{\delta_{k}}\right)^{m}\right|
\end{aligned}
$$

If we sum this last expression first with respect to $m$ and use the fact that $|z|<\gamma_{n_{0}+n}$, we get

$$
\begin{aligned}
\sum_{k=n_{0}+n}^{n_{0}+n+p} \frac{|z|}{\gamma_{n_{0}+n}-|z|} & -\frac{|z| \gamma_{k}}{\gamma_{n_{0}+n} \delta_{k}-|z| \gamma_{k}} \\
& =\sum_{k=n_{0}+n}^{n_{0}+n+p} \frac{|z| \gamma_{n_{0}+n}\left(\frac{\epsilon_{k}}{\gamma_{k}}\right)}{\left(\gamma_{n_{0}+n}-|z|\right)\left(\gamma_{n_{0}+n}\left(1+\frac{\epsilon_{k}}{\gamma_{k}}\right)-|z|\right)} \\
& \leqq \frac{|z|}{\left(\gamma_{n_{0}+n}-|z|\right)^{2}} \sum_{k=n_{0}+n}^{n_{0}+n+p} \epsilon_{k} .
\end{aligned}
$$


Then if we choose $N(\epsilon)$ so large that

$$
\sum_{k=N(\epsilon)}^{\infty} \epsilon_{k}<\frac{\left(\gamma_{n_{0}+1}-r_{0}\right)^{2}}{r_{0}} \epsilon
$$

the uniform convergence of the original series in $\left\{|z| \leqq r_{0}\right\}$ is established.

$$
\text { Lemma 6. } \pi(z)=f(z) \text { for } z \in\{|z|<r\} \text {. }
$$

Proof. Because of Lemma 4 , there exists $\rho>0$ such that $R_{n}(z) / z \neq 0$ and $\pi(z) / z \neq 0$ for $|z|<\rho$. For $m \geqq 1$,

$$
\begin{aligned}
0 & <\sum_{k=i}^{\infty}\left(\frac{1}{\gamma_{k}^{m}}-\frac{1}{\delta_{k}^{m}}\right)=\sum_{k=i}^{\infty} \frac{\delta_{k}^{m}-\gamma_{k}^{m}}{\gamma_{k}^{m} \delta_{k}^{m}} \\
& \leqq \frac{1}{\gamma_{i}^{m}} \sum_{k=i}^{\infty}\left(1-\left(\frac{\gamma_{k}}{\delta_{k}}\right)^{m}\right) \\
& =\frac{1}{\gamma_{i}^{m}} \sum_{k=i}^{\infty}\left[m \frac{\epsilon_{k}}{\delta_{k}}-\frac{m(m-1)}{2}\left(\frac{\epsilon_{k}}{\delta_{k}}\right)^{2}+\cdots \pm\left(\frac{\epsilon_{k}}{\delta_{k}}\right)^{m}\right] \\
& =\frac{1}{\gamma_{i}^{m}} \Phi(i)
\end{aligned}
$$

where $\lim _{i \rightarrow \infty} \Phi(i)=0$. Similarly,

$$
0<\sum_{k=i}^{n}\left(\frac{1}{\gamma_{k, i}^{m}}-\frac{1}{\delta_{k, i}^{m}}\right) \leqq \frac{1}{\gamma_{i, n}^{m}} \psi_{n}(i)
$$

where $\lim _{i \rightarrow \infty}\left(\lim _{n \rightarrow \infty} \psi_{n}(i)\right)=\lim _{i \rightarrow \infty} \psi(i)=0$. Therefore,

$$
\begin{aligned}
0 & \leqq \limsup _{n \rightarrow \infty}\left|\sum_{k=2}^{n}\left[\frac{1}{\gamma_{k, n}^{m}}-\frac{1}{\delta_{k, n}^{m}}\right]-\sum_{k=2}^{\infty}\left[\frac{1}{\gamma_{k}^{m}}-\frac{1}{\delta_{k}^{m}}\right]\right| \\
& \leqq \limsup _{n \rightarrow \infty}\left|\sum_{k=n_{0}}^{n}\left[\frac{1}{\gamma_{k, n}^{m}}-\frac{1}{\delta_{k, n}^{m}}\right]-\sum_{k=n_{0}}^{\infty}\left[\frac{1}{\gamma_{k}^{m}}-\frac{1}{\delta_{k}^{m}}\right]\right| \\
& \leqq \lim _{n \rightarrow \infty}\left(\frac{1}{\gamma_{n_{0}, n}^{m}} \psi_{n}\left(n_{0}\right)+\frac{1}{\gamma_{n_{0}}^{m}} \Phi\left(n_{0}\right)\right) \\
& =\frac{1}{\gamma_{n_{0}}^{m}}\left(\psi\left(n_{0}\right)+\Phi\left(n_{0}\right)\right) .
\end{aligned}
$$

Since $\lim _{n_{0} \rightarrow \infty} \psi\left(n_{0}\right)+\Phi\left(n_{0}\right)=0$ and $\lim _{n_{0} \rightarrow \infty} \gamma_{n_{0}}$ is $r>0$,

$$
\limsup _{n \rightarrow \infty}\left|\sum_{k=2}^{n}\left(\frac{1}{\gamma_{k, n}^{m}}-\frac{1}{\delta_{k, n}^{m}}\right)-\sum_{k=2}^{\infty}\left(\frac{1}{\gamma_{k}^{m}}-\frac{1}{\delta_{k}^{m}}\right)\right|=0 .
$$


Thus all the coefficients of the Taylor series expansion of $\log \left(R_{n}(z) / \pi(z)\right)$ about $z=0$ can be shown to have a limit zero as $n \rightarrow \infty$, and this $\lim _{n \rightarrow \infty} \log \left\{R_{n}(z) / \pi(z)\right\}=0$ which implies that $\lim _{n \rightarrow \infty} R_{n}(z)=\pi(z)=f(z)$.

Remarks. It is clear that the specific form of the cuts was not essential in the construction of the uniformizing function. For this reason it is clear that the conditions on the cuts could be relaxed to permit variation in length and shape provided that they are symmetric, the resulting surface is hyperbolic, and the zeros and poles lie (except for a finite number of cases) on one side of the origin.

\section{BIBLIOGRAPHY}

1. H. B. Curtis, Jr., Some properties of functions which uniformize a class of simply connected Riemann surfaces, Proc. Amer. Math. Soc. 10 (1959), 525-530.

2. - The uniformization of a class of simply connected Riemann surfaces, Proc. Amer. Math. Soc. 11 (1960), 511-516.

3. G. R. MacLane, Riemann surfaces and asymptotic values associated with real entire functions, The Rice Institute Pamphlet, Houston, Texas, 1952.

4. H. E. Taylor, Determination of the type and properties of the mapping functions of a class of doubly connected Riemann surfaces, Proc. Amer. Math. Soc. 4 (1953), 52-68.

Defense Research Laboratory, The University of Texas 